and perivascular lymphocytic infiltrates were almost universal, but probably secondary inflammation from chronic rubbing of the eyes and not pathogenetic. Many examples of congenital absence of specific striated muscles throughout the body are known and congenital ptosis is another. Isolated absence of smooth muscles is rarer. The upper eyelid is one of only a few sites in the body where smooth and striated muscle must work together for function, the absence of one not fully compensated by the other.

\section{Abstract A18}

Lissencephaly and circumferential skin creases associated with TUBB mutation broaden the spectrum of tubulinopathies

C. Fallet-Bianco ${ }^{1}$, S. Boissel ${ }^{2}$, F. Rypens ${ }^{1}$, D. Bouron-Dal Soglio $^{1}$, J. Michaud ${ }^{2}$

${ }^{1}$ CHU Sainte-Justine-Université de Montréal, Montréal (QC), Canada; ${ }^{2} \mathrm{CHU}$ Sainte-Justine Research Center, Université de Montréal, Montréal (QC), Canada

doi:10.1017/cjn.2017.19

Mutations in tubulin genes cause cortical malformations, rarely with minor dysmorphic features. Congenital circumferential skin creases are rare disorders characterized by ring creases associated with facial dysmorphism, intellectual disability and imaging brain data from normal to malformations involving corpus callosum and vermis. The cause was unknown until recent data demonstrated that mutations in TUBB are responsible for this syndrome for which neuropathological data have never been described.

A termination of pregnancy was performed at $28 \mathrm{WG}$ for brain malformations. Karyotype was normal and whole-exome sequencing was performed for subject and parents. Examination disclosed severe dysmorphic features, circumferential creases and microcephaly. Neuropathological study demonstrated microlissencephaly, callosal agenesis, dysmorphic basal ganglia, cerebellar hypoplasia. Histological examination showed cortical glomerular structures, abnormal cortico-spinal tracts, heterotopic axonal fascicles, unusually large germinal zones, abnormal hippocampi, roughly-shaped dentate and olivary nuclei. Whole-exome sequencing demonstrated a heterozygous missense mutation in TUBB gene occurring de novo.

Neuropathological features are identical to those observed in other tubulinopathies. However, mutations in TUBB gene have not yet been reported in tubulinopathies with isolated cortical malformations. The association of circumferential skin creases, facial dysmorphism and a characteristic brain malformation resulting from a mutation in TUBB gene constitutes a new entity expanding the spectrum of tubulinopathies.

\section{Abstract A19}

The neuropathology of the brain malformation in fetal PI3KR2 related disease

P. Diamandis ${ }^{1}$, S. Keating ${ }^{2}$, D. Chitayat ${ }^{2}$, G. Mirzaa $^{3}$, P. Shannon ${ }^{4}$

${ }^{1}$ University Health Network, Toronto, Ontario; ${ }^{2}$ Mount Sinai Hospital, Toronto, Ontario; ${ }^{3}$ Seattle Children's Research Institute,
Seattle, Washington; ${ }^{4}$ Mount Sinai Hospital, Toronto, Ontario, Canada

doi:10.1017/cjn.2017.20

Activating germline mutations in PI3KR2 (Phosphatidylinositol 3-kinase regulatory subunit 2) have been associated with a syndrome of macrencephaly, polydactyly and Polygyria (MIM\#603387), which is well described in the clinical and radiological literature not histologically. We present the first pathological description of the condition of which we are aware in a 20 week gestation fetus. Midgestation ultrasound demonstrated complex congenital heart disease, and the pregnancy was interrupted at 20 weeks gestation. Neuropathological examination demonstrated cerebral macroencephaly, with a weight greater than twice that expected for gestational age. The hemispheres were symmetrically swollen with blunted Sylvian fissures, mildly enlarged lateral ventricles and thickened cerebral mantles. Histology demonstrated leptomeningeal and subcortical heterotopia, as well as premature and abnormal neocortical lamination, principally in the frontal lobes. Cajal Retzius cells displayed enlarged Reelin $(+)$ varicosities extending into the superficial cellular layers of the cortex, and layer II demonstrated a population of large pyramidal cells. An intracortical calretinin (+) hypocellular band was sometimes present.

\section{Titles of Diagnostic Case Presentations}

\section{Atypical teratoid/rhabdoid tumour of the sella turcica}

R. J. B. Macaulay

Department of Anatomic Pathology, Moffitt Cancer Center, Tampa, FL

\section{Cortical ependymoma presenting with long - term} refractory epilepsy

\author{
H. Alghefari, B AlYamany, LC Ang \\ London Health Science Center Ontario, Canada
}

\section{Osmotic Demyelination Syndrome secondary to recurrent hypoglycemia}

\section{$J$. Karamchandani and R. Auer}

Department of Pathology, McGill University, Montreal, QC;

Department of Pathology, University of Saskatchewan, Saskatoon, Saskatoon, SK

\section{Amyloid beta-related angiitis}

S. Magaki, S. Karnezis, L. Macyszyn, and H. V. Vinters

UCLA Medical Center, Los Angeles, CA

\section{Intravascular large B cell lymphoma}

L.M. Schmitt and L. Resch

Division of Neuropathology, University of Calgary, Calgary, Alberta 
6. Disseminated (malignant) non-Langerhan histiocytosis of CNS

Qi Zhang and Lee Cyn Ang

Department of Pathology, Western University, London, Ontario

\section{CNS blastomycosis and aspergillosis}

S. Krawitz, B. Mansouri, R. Rivera, J. Safneck

Department of Pathology, University of Manitoba, Winnipeg, MB

8. Myoepithelioma (parachordoma); EWSR1 and FUS rearrangement negative

Viktor Skihar, Claire I. Coiré, Dennis Izukawa
Department of Pathology, Sunnybrook Health Science Centre, Toronto, ON;

Departments of Pathology, Trillium Health Partners, Mississauga, ON

9. Congenital hypothalamic hamartoma in context of Pallister Hall Syndrome

C. Dunham, D. McFadden, L. Dahlgren, B. Butler, S.Hamilton, M. McKinnon

Children and Women's Health Centre of BC 